\title{
Viewing behavior: Ocular and attentional disengagement
}

\author{
WA JAMES TAM and LEW B. STELMACH \\ Communications Research Centre, Ottawa, Ontario, Canada
}

\begin{abstract}
In five experiments, we examined the role of the ocular and attentional systems in determining saccadic latencies. Prior to making a saccade to a target stimulus, subjects were required to direct their attention to a foveal stimulus or to an eccentric stimulus. Either stimulus could be extinguished before the onset of the target. Saccadic latencies were shortest when the foveal stimulus was extinguished, regardless of whether it was attended or not. Control experiments showed that subjects were able to attend properly and that warning, arising from turning off a stimulus before target onset, could not completely account for the results. The results were discussed in terms of ocular disengagement, attentional disengagement, and joint ocular-attentional disengagement. It was concluded that an explanation emphasizing ocular disengagement provided the best account of the data.
\end{abstract}

Viewing a visual scene involves a sequence of fixations and saccades to different parts of the scene. This process of sampling the scene consists of engagement of the eyes on a feature of interest, a period of information acquisition, disengagement of the eyes, and movement of the eyes toward a new stimulus (Reuter-Lorenz, Hughes, \& Fendrich, 1991; Tam, 1989; Tam \& Ono, 1984, 1988).

It is also possible to sample a scene without eye movements (Engel, 1971; Eriksen \& Hoffman, 1972; Helmholtz, cited in Groner \& Groner, 1989; Jonides, 1980; Kaufman \& Richards, 1969; Posner, 1980; Wundt, cited in Groner \& Groner, 1989). This can be accomplished by an attentional system, often referred to as covert attention, spatially directed attention, or an attentional spotlight (LaBerge \& Brown, 1989; Posner, Snyder, \& Davidson, 1980). Attentional sampling can be characterized in much the same way as ocular sampling, in that attention is engaged on a feature of interest and is held there for a period of time, during which information is acquired, disengaged, and, eventually, directed to a new feature (Braun \& Breitmeyer, 1988; Fischer, 1987; Mayfrank, Mobashery, Kimmig, \& Fischer, 1986; Posner, 1980). Although attentional sampling can operate independently of ocular sam-

This research was supported by the Communications Research Centre, Federal Government of Canada, and by a grant from the Natural Sciences and Engineering Research Council of Canada to L.B.S. We gratefully acknowledge the assistance of Rod McNeil and Greg Craig in the data collection. Vince Di Lollo and John Logan provided equipment for measuring EOGs in Experiment 5. We thank Paul Hearty for support throughout the course of this project and for helpful comments on an earlier version of this paper. Ted Grusec, Chris Herdman, Tom Whalen, and two anonymous reviewers also helped improve this paper with valuable suggestions. Finally, we thank Angela Quang and Vera Yuzyk for support throughout the course of the project. The authors can be contacted at the Communications Research Centre, 3701 Carling Avenue, Ottawa, Ontario, Canada K2H 8S2 (e-mail: james@dgbt.doc.ca and lew@dgbt.doc.ca). pling, the two systems normally operate in concert (e.g., Klein, 1980; Posner, 1980; Remington, 1980; Shepherd, Findlay, \& Hockey, 1986).

Many interesting issues arise when one considers the joint functioning of ocular and attentional sampling. The issue we addressed in the present paper concerns the role of ocular and attentional disengagement in determining saccadic latencies. Three distinct possibilities were examined: Saccadic latencies are determined predominantly by the ocular sampling system, predominantly by the attentional sampling system, or jointly by the ocular and attentional sampling systems.

In the modal technique for studying saccadic latencies, subjects fixate centrally and execute a saccade to a target that appears in the periphery. Research using this technique has shown that a saccade to a target can be initiated sooner if the foveal stimulus is turned off than if the foveal stimulus is left on (Becker, 1972; Cohen \& Ross, 1977; Fischer, 1987; Fischer \& Boch, 1983; Fischer \& Ramsperger, 1984; Hallett \& Adams, 1980; Mayfrank et al., 1986; Reulen, 1984; L. Ross \& S. Ross, 1980; S. Ross \& L. Ross, 1981, 1983; Saslow, 1967; Wenban-Smith \& Findlay, 1991). One interpretation of this finding is that the ocular sampling system needs input to remain in an engaged state, and that turning off the stimulus at the fixation point leads to disengagement of the ocular system. When the new eccentric stimulus appears, the ocular system is already disengaged and, therefore, the latency to initiate the saccade is shorter (Reuter-Lorenz et al., 1991; Tam, 1989; Tam \& Ono, 1984, 1988). This interpretation emphasizes the role of the ocular sampling system, and explains the shortened saccadic latencies using strictly ocular processes.

Another interpretation emphasizes the role of the attentional sampling system (Braun \& Breitmeyer, 1988; Fischer, 1987; Fischer \& Breitmeyer, 1987; Mayfrank et al., 1986). According to the attentional interpretation, 
a saccade must be preceded by attentional disengagement. Turning off the stimulus at the fixation point leads to disengagement of the attentional system, thereby promoting a rapid shift of the eyes to the new stimulus.

The attentional interpretation is supported by empirical findings obtained with a dissociation paradigm, in which subjects attended to an eccentric stimulus, fixated on a stimulus at the center of a display, and made a saccade toward a new target stimulus. Latencies of saccades toward the target were shorter when the attended stimulus was turned off before the appearance of the target than when it was left on. This was so, even though the central foveal stimulus remained unchanged. This finding led researchers to conclude that saccadic latencies were determined by the attentional, and not the ocular, sampling system (Braun \& Breitmeyer, 1988; Mayfrank et al., 1986). This conclusion was based on the assumptions that foveating the central fixation stimulus and attending to the eccentric stimulus engaged the ocular and attentional sampling systems, respectively. Turning off the eccentric stimulus promoted disengagement of the attentional system, leaving the ocular system in an engaged state. When the target appeared, the eye could immediately move because the attentional system did not need to be disengaged. One limitation of this work was that it did not assess the contribution of ocular disengagement by extinguishing the unattended foveal stimulus.

In the present work, we examined the contribution of ocular and attentional disengagement to saccadic latencies within the same series of experiments. The ocular system was engaged by having subjects foveate a central stimulus, and the attentional system was engaged by having them attend to an eccentric stimulus; on a given trial, the subjects fixated centrally and attended eccentrically. Ocular disengagement was promoted by turning off the foveal stimulus, and attentional disengagement was promoted by turning off the attended stimulus.

In Experiment 1, we measured saccadic latencies to target onset while varying the temporal relationship between target onset and the offset of the foveal or the eccentric stimulus. In Experiment 2, we determined whether the findings with eye movements would generalize to manual responses. In Experiment 3, we implemented better attentional manipulations, making it easier for the subjects to attend properly. In Experiment 4, we measured whether the subjects had indeed allocated attention properly during each trial. In Experiment 5, we measured saccadic latencies with finer temporal resolution in order to assess the qualitative aspects of the latency distributions. In Experiments 4 and 5 , we also included a condition in which the subjects fixated and attended centrally. The experiments are reported in the order they were conducted, except for Experiments 1 and 2, which were run concurrently.

\section{EXPERIMENT 1}

The purpose of Experiment 1 was to examine the contribution of ocular and attentional disengagement to sac- cadic latencies. The subjects were presented with a display that consisted of two dots (see Figure 1). They were required to foveate the central dot and to direct their attention covertly toward the eccentric dot that was located below. Their task was to make a saccade to a third, target dot, which appeared either to the left or to the right of the foveal dot. The interval between the offset of one of the first two dots and the onset of the target dot was varied systematically. This interval is referred to as the offset-onset asynchrony. By convention, overlap conditions refer to intervals in which the target appeared when the attended eccentric or the foveal stimulus was still on. Conversely, gap conditions refer to intervals in which the target appeared after the attended or the foveal stimulus was turned off.

According to the ocular disengagement hypothesis, latencies of saccades should be shorter when the ocular sampling system is in a disengaged state. This should occur in gap conditions when the foveal stimulus is turned off. According to the attentional disengagement hypothesis, latencies of saccades to a target should be shorter when the attentional sampling system is in a disengaged state. This should occur in gap conditions when the attended stimulus is turned off. Finally, according to the hypothesis that both ocular and attentional sampling systems play a role, turning off either stimulus should lead to shorter saccadic latencies.

\section{Method}

Subjects. The two authors and 2 paid naive subjects participated in the experiment. All the subjects were male and were between 25 and 40 years of age. The naive subjects were recruited from the population at large and had never participated in a psychophysical experiment.

Stimuli and Apparatus. The display was presented on a Tektronix 608 oscilloscope equipped with P15 phosphor and was controlled by an Interactive Electronic Systems controller (Finley, 1985). The P15 phosphor has an extremely short persistence and decays to $10 \%$ of its original value in $2 \mu \mathrm{sec}$. The background luminance of the display was $50 \mathrm{~cd} / \mathrm{m}^{2}$ and the luminance of the stimuli was $400 \mathrm{~cd} / \mathrm{m}^{2}$.

The display consisted of a sequence of panels, as shown in Figure 1. At the beginning of each trial two dots were displayed, one at the center of the oscilloscope screen and the other $3^{\circ}$ below the center. These are labeled "foveal" and "eccentric," respectively, in Figure 1. The overlap and gap conditions differed as to where in the sequence of panels the offset of the foveal or the eccentric dot occurred. In the overlap conditions the offset occurred in the final display, whereas in the gap conditions the offset occurred in the intermediate display. The target consisted of a dot added to the intermediate display randomly to the left or the right of the screen. In Figure 1, the target appears to the right.

A headrest and chinrest were used to help maintain a steady head position. Eye position was monitored at $60 \mathrm{~Hz}$ with a pupil-tracking system (ISCAN RK-416), and thus saccadic latency was measured with a resolution of $16.7 \mathrm{msec}$. The spatial resolution of the eye tracker was better than $0.25^{\circ}$. The equipment was controlled by an 80386-based computer.

Design. There were two factors in the experiment. The first was location of offset, referring to the stimulus that was turned off (foveal or eccentric). Only one was turned off in any given trial. The second factor was offset-onset asynchrony $(-500,0,200$, and $500 \mathrm{msec}$ ), referring to the temporal interval between the offset of 


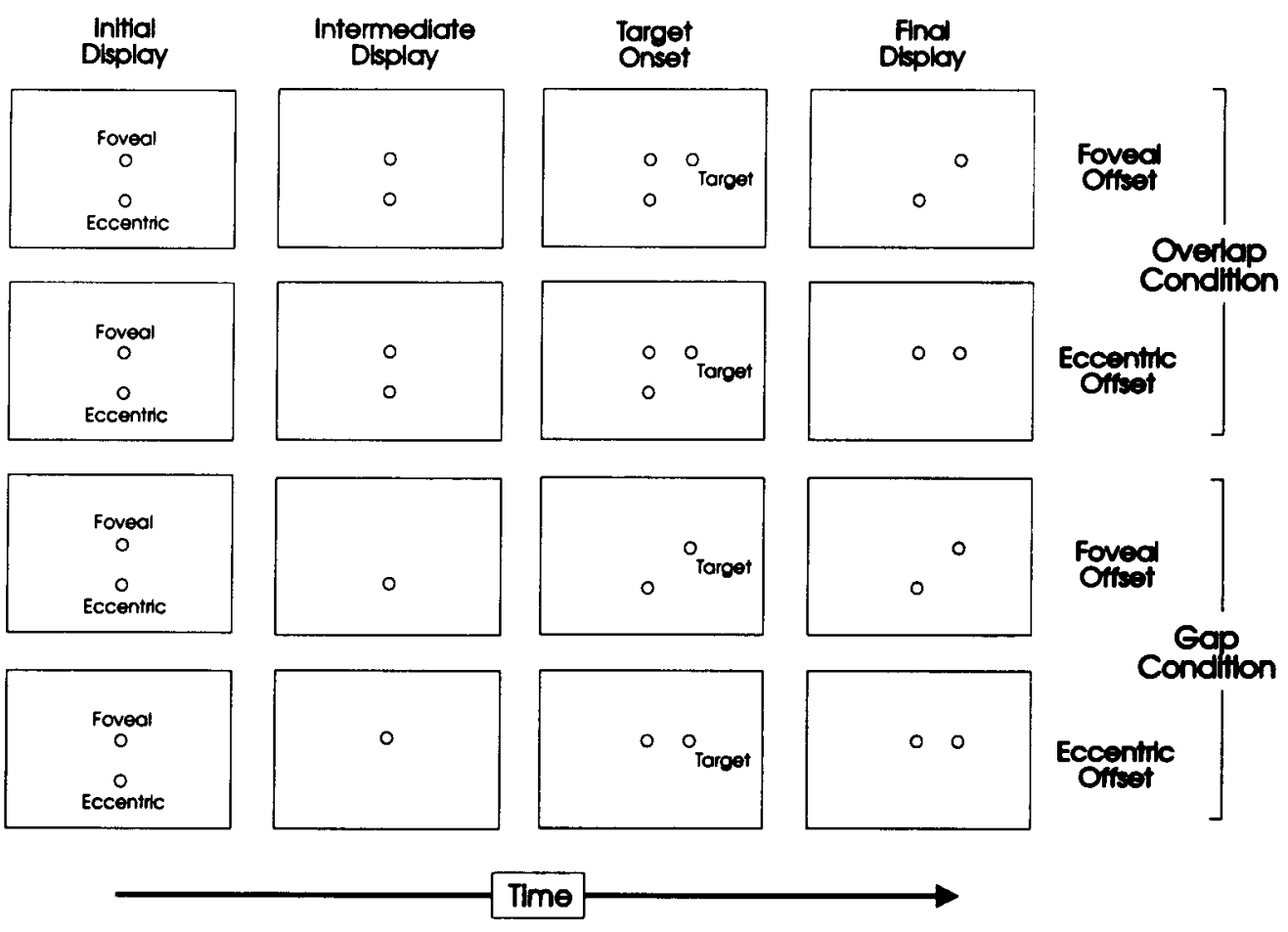

Figure 1. Schematic representation of stimulus configurations and sequences. In Experiments 1 and 2, the eccentric stimulus was always below the foveal stimulus. In Experiments 3, 4, and 5, the eccentric stimulus was a picture rather than a dot, and alternated above or below the foveal stimulus from trial to trial.

the foveal stimulus or the offset of the eccentric stimulus and the onset of the target. The negative offset-onset asynchrony of $-500 \mathrm{msec}$ indicated that the foveal and eccentric dots were still present when the target appeared (overlap condition). The positive asynchronies of 200 and $500 \mathrm{msec}$ indicated that either the foveal dot or the eccentric dot was turned off before the onset of the target dot (gap condition). At a 0 -msec offset-onset asynchrony, the offset of one of the dots in the initial display occurred simultaneously with the onset of the target and there was no intermediate display. The offset-onset asynchronies used in the present experiment were selected because they are believed to be associated with saccadic latencies when the visual system is engaged $(-500 \mathrm{msec})$, in intermediate states ( 0 and $200 \mathrm{msec}$ ), and disengaged (500 msec) (e.g., Braun \& Breitmeyer, 1988). Thus, there were eight experimental conditions ( 2 locations of offset $\times 4$ asynchronies).

Each condition was tested 40 times per subject over the course of four sessions. There were 80 trials per session. In each trial the condition was chosen randomly. The subjects completed one to two sessions per day. Sessions from this experiment were alternated with sessions from Experiment 2, in which a manual response to the target was required rather than a saccade. Alternate sessions in the saccadic and manual tasks helped equalize possible practice effects. The order of the two tasks was counterbalanced among subjects.

Procedure. The subjects viewed the display $46 \mathrm{~cm}$ away from the oscilloscope screen. Each session began with a brief calibration of the eye tracker. The experimental trials began immediately after calibration.

At the beginning of each trial, two dots appeared on the screen (initial display in Figure 1). The subjects foveated the central dot and focused their attention on the lower dot. A trial began after a random interval of 1.2-1.7 sec. At the negative offset-onset asynchrony ( $-500 \mathrm{msec}$, overlap condition), the target dot appeared first, followed by the offset of one of the initial dots. At the positive offset-onset asynchronies (200 and $500 \mathrm{msec}$, gap conditions), the onset of the target dot followed the offset of one of the initial dots. In all conditions, the subjects responded as quickly as possible with a saccade to the target. The trials were automatically initiated by the computer. The intertrial interval was varied randomly between 1.5 and $2.3 \mathrm{sec}$. When the subjects needed a rest, they could stop the initiation of a new trial by pressing a button, and they could resume the trials by pressing the button again. Each session lasted about $15 \mathrm{~min}$.

Saccade detection. Saccades were detected by using an amplitude criterion. A saccade was indicated when eye position deviated $0.3^{\circ}$ from a baseline; baseline was the average eye position just prior to target onset. This amplitude criterion is equivalent to a velocity criterion of about $20^{\circ} / \mathrm{sec}$, as used by other researchers (e.g., Hallett \& Adams, 1980).

The time at which a saccade was detected indicated the point at which the subject's eyes were just beginning to move toward the target. The temporal interval between the onset of the target and the detection of the saccade is referred to as the saccadic latency.

Rejection criteria. A trial was rejected if a blink or an eye movement occurred before target onset or if the eye movement was in the wrong direction. A trial was also rejected if the latency of the saccade was either less than $100 \mathrm{msec}$ or greater than $600 \mathrm{msec}$. Latencies outside this range were considered to be anticipatory or excessively delayed (Findlay, 1981; Kalesnykas \& Hallett, 1987). Rejected trials, which accounted for less than $5 \%$ of the total number of trials averaged over the 4 subjects, were repeated in the same session.

\section{Results and Discussion}

The results of Experiment 1 are shown graphically in the top panel of Figure 2, averaged over all 4 subjects. Filled triangles show the results for the conditions in which the eccentric dot was turned off and open circles show the 

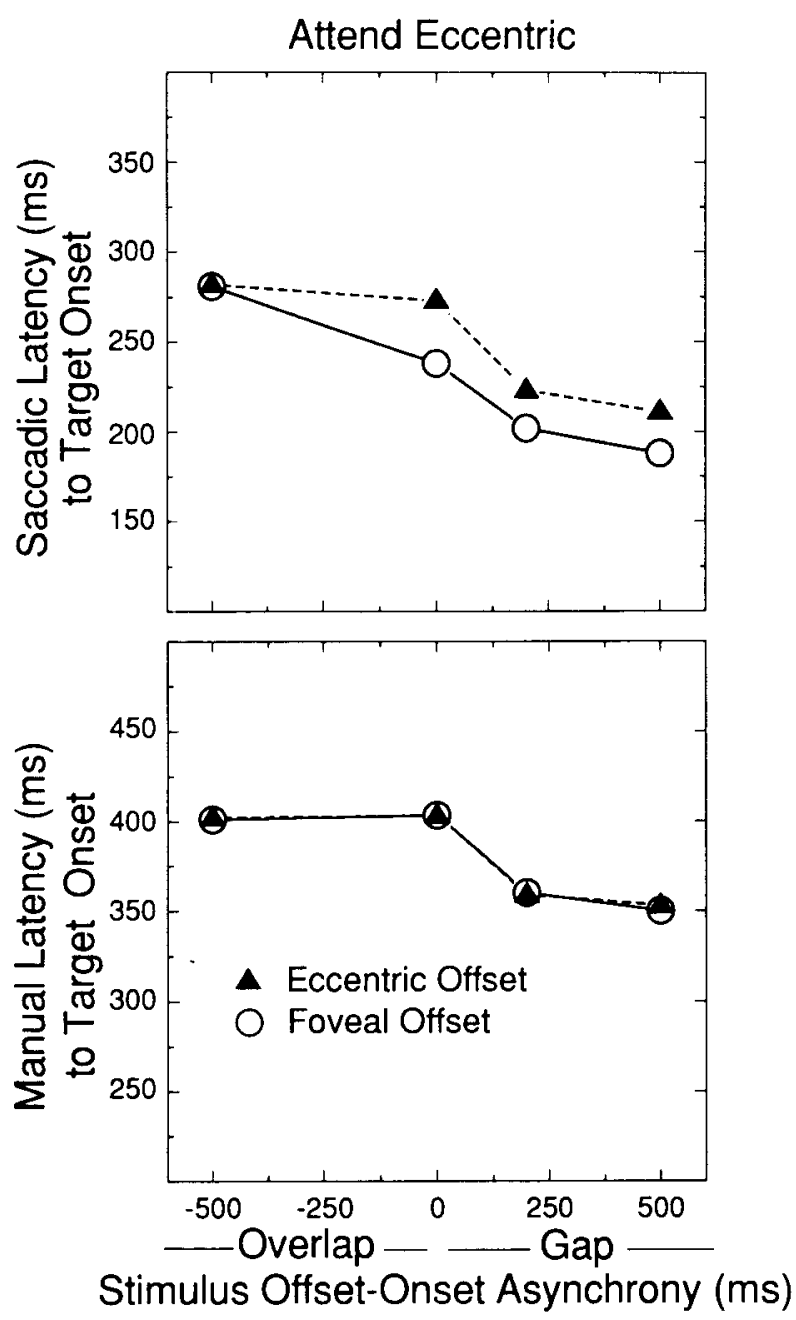

Figure 2. Saccadic latencies (from Experiment 1) and manual latencies (from Experiment 2) are shown as a function of asynchrony between stimulus offset and target onset. Each point in the figure represents the mean of the data for 4 subjects.

results for the conditions in which the foveal dot was turned off. These symbols are also used in Figures 2, 3, and 4.

The -500 -msec offset-onset asynchrony provided a baseline condition for measuring the change in saccadic latency occurring at other asynchronies. In this condition, the target dot appeared on the screen when both the foveal and the attended eccentric dots were still visible and, therefore, both the ocular and attentional sampling systems were in an engaged state. As might be expected at this asynchrony, mean saccadic latencies were equivalent for both foveal and eccentric offsets $(282 \mathrm{msec})$.

At other offset-onset asynchronies (above $-500 \mathrm{msec}$ ), there were two major aspects to the results. First, saccadic latencies decreased regardless of the stimulus that was turned off. Second, saccadic latencies decreased more when the unattended foveal stimulus was extinguished than when the attended eccentric stimulus was extinguished.
A 2 (location of offset) $\times 4$ (offset-onset asynchrony) within-subject analysis of variance confirmed the above observations. The main effects of location of offset $[F(1,3)=15.27, p<.05]$ and offset-onset asynchrony $[F(3,9)=32.50, p<.01]$, as well as the interaction of location with offset-onset asynchrony $[F(3,9)=5.73$, $p<.05]$ were statistically significant.

The shortened latencies in the gap conditions, in which the attended eccentric stimulus was turned off (filled triangles, top panel of Figure 2), are consistent with the attentional disengagement hypothesis. According to this hypothesis, turning off the attended eccentric stimulus promoted disengagement of the attentional sampling system, thereby reducing saccadic latencies. This aspect of the results concurs with Braun and Breitmeyer (1988) and Mayfrank et al. (1986).

In a similar manner, the shortened saccadic latencies in the unattended foveal-offset condition (open circles, top panel of Figure 2) suggest a role for ocular disengagement. According to the ocular disengagement hypothesis, turning off the foveal stimulus promoted disengagement of the ocular sampling system, thereby reducing saccadic latencies. The argument for a role for ocular disengagement is especially compelling because the magnitude of the gap effect was larger when the unattended foveal stimulus was extinguished than when the attended eccentric stimulus was extinguished. In the top panel of Figure 2, note that the open circles lie below the filled triangles for gap conditions.

In summary, the results of Experiment 1 are consistent with the view that both ocular and attentional disengagement play a role in determining saccadic latencies. However, it would be valuable to demonstrate that the effects are associated with saccadic-related processes and do not reflect a general facilitatory alerting due to warning caused by offsets (e.g., Ross \& Ross, 1980). Specifically, if the effects were to generalize to other response modalities, it might be inappropriate to attribute them to ocular and attentional disengagement.

\section{EXPERIMENT 2}

A manual response was used in this experiment, in order to determine if the pattern of results in the previous experiment would generalize to a manual response task. For example, if the greater decrease in the foveal-offset condition was due to ocular disengagement, we would not expect the effect to generalize to the manual task because the execution of a manual response is not contingent upon ocular disengagement.

\section{Method}

The subjects, the display, and the procedure were the same as in Experiment 1, with the exception that the subjects did not make a saccade to the target, but instead made a manual response (measured with a resolution of $0.1 \mathrm{msec}$ ). The subjects were instructed to press a left or a right button as quickly as possible to indicate whether the left or the right target had appeared, respectively. They were also instructed not to move their eyes during a trial. As men- 
tioned previously, sessions in Experiment 2 were alternated with sessions in Experiment 1. The average number of rejected trials was less than $5 \%$.

\section{Results and Discussion}

In contrast to Experiment 1, manual latencies in the gap conditions did not depend on the location of the offsets. Note that for gap conditions, the open circles are superimposed on the filled triangles in the bottom panel of Figure 2 (Experiment 2 ) but that the open circles lie below the filled triangles in the top panel of Figure 2 (Experiment 1). This pattern of results suggests that the interaction observed in Experiment 1 was a uniquely ocular effect, because it did not generalize to the manual task. Bear in mind that the experiments were run concurrently, used the same subjects, and were identical in all respects except for the response task.

Nevertheless, the shortened manual latencies in the gap conditions $[F(3,9)=59.83, p<.01]$ suggest a common component to the gap effect. For example, the offset (foveal or eccentric) that preceded the target in the gap conditions may have warned the subjects of the impending target, resulting in both shorter manual latencies and shorter saccadic latencies (e.g., Ross \& Ross, 1981).

The presence of a warning effect in the current experiment compels us to consider the role of warning in explaining the results of Experiment 1. In the extreme case, the entire decrease in saccadic latencies produced by the attended eccentric offset might have been due to warning, and the additional decrease for the foveal offset might have been due to disengagement of the ocular sampling system. In a more moderate view, only a portion of the decrease produced by the attended eccentric offset need be attributed to visual warning. In any case, the evidence supporting a role for attentional disengagement in shortening saccadic latencies is difficult to disambiguate from visual warning. In contrast, the evidence favoring a role for ocular disengagement is clear and compelling. Warning cannot explain the additional decrease produced by the foveal offset in Experiment 1.

\section{EXPERIMENT 3}

A key assumption of the present research was that the subjects were able to dissociate their direction of attention from their direction of fixation. If this assumption were false, then the interpretation of the experiments would be problematic. Specifically, it might be possible to explain the shorter saccadic latencies in the foveal-offset conditions by assuming that the foveated location always received more attention, despite instructions to attend to the eccentric location. To ensure that the subjects attended properly, we replicated Experiments 1 and 2 with stimuli that made it easier for them to maintain attention at the eccentric location. In Experiment 3, the eccentric stimulus was made more interesting by using pictures of objects instead of a dot. The pictures were also larger than the fixation stimulus, which was still a dot. To further ensure that the sub- jects were attending properly, the location of the eccentric stimulus was alternated above and below the fixation point from trial to trial. The subjects initiated each trial when they were confident that they were attending properly.

\section{Method}

Subjects. Five subjects, including the two authors and 3 paid subjects who were naive as to the experimental hypotheses, participated in the experiment. Of the 3 naive subjects, 2 had not participated in Experiment 1. The 2 new subjects were experienced in psychophysical experiments and were recruited from a nearby university community. The ages of the subjects ranged from 25 to 40 years.

Stimuli and Apparatus. Aside from a change in the stimuli, the arrangement of the stimuli on the screen and the apparatus were the same as those described in Experiment 1. Instead of a dot, the attended eccentric stimulus was a picture-a line drawing of a duck, a dog, a horse, a watercan, or a sailboat. The picture was drawn within an area subtending an angle of $1.4^{\circ}$ square and was located $3^{\circ}$ above or below the central dot, as measured from the center of the picture to the center of the foveal dot.

Design. There were two factors-location of offset (foveal or eccentric) and offset-onset asynchrony $(-500,-100,0,100,150$, 200,300 , and $500 \mathrm{msec}$ ) - for a total of 16 experimental conditions. There were 64 trials per session. Each condition was tested four times in a session and each subject served for 10 sessions. As before, there were $\mathbf{4 0}$ observations per experimental condition.

Procedure. The procedure was the same as in Experiment 1, with the following exceptions. The subjects initiated each trial with a buttonpress rather than the computer initiating the trials automatically. The picture was randomly selected from the set of five pictures and was displayed, on alternate trials, $3^{\circ}$ above or below the foveal dot. The subjects performed saccadic or manual responses on alternate sessions. Rejected trials due to misfixation, too fast or too slow a response, or noise in the monitoring system accounted for less than $8 \%$ of the trials. Rejected trials were repeated in the same session.

\section{Results and Discussion}

Figure 3 shows the results of Experiment 3, averaged over all 5 subjects. As before, the overlap condition of -500 -msec offset-onset asynchrony provided a baseline condition against which saccadic or manual latencies at other asynchronies could be compared. In the baseline condition, latencies were similar for both foveal and eccentric offsets, regardless of the response task.

The major aspects of Experiments 1 and 2 were replicated. First, there was a decrease in both saccadic and manual latencies in the gap conditions. Second, saccadic latencies decreased more when the unattended foveal stimulus was extinguished than when the attended eccentric stimulus was extinguished. Note that the open circles lie below the filled triangles in the top panel of Figure 3 . Third, the manual latencies for attended eccentric and foveal offsets were not significantly different from each other. Note that the open circles and filled triangles lie on the same curve in the bottom panel of Figure 3.

Saccadic and manual latencies were analyzed separately. A 2 (location of offset) $\times 8$ (offset-onset asynchrony) within-subject analysis of variance showed that the effects evident in Figure 3 were statistically significant. The analysis for saccadic latencies showed that the main effects of location of offset $[F(1,4)=33.26, p<.01]$ and off- 

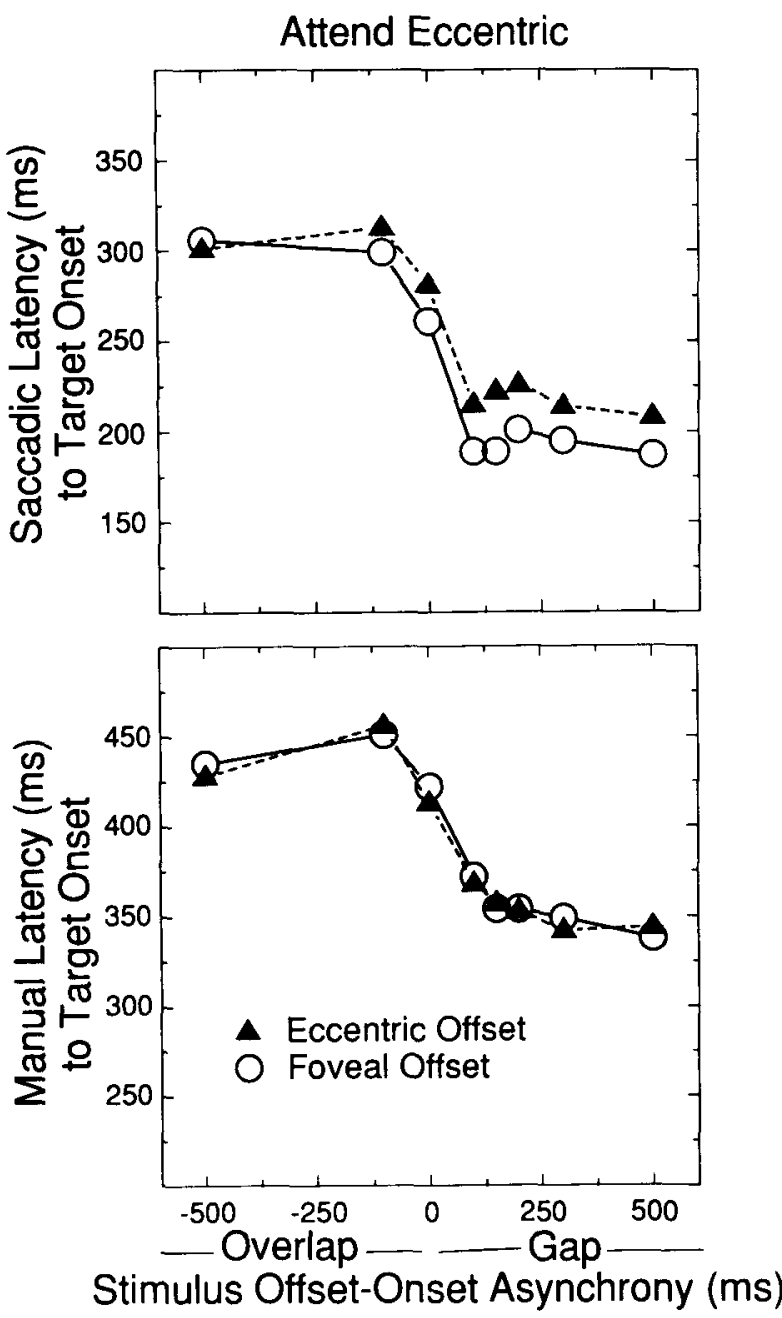

Figure 3. Saccadic and manual latencies (from Experiment 3) are shown as a function of asynchrony between stimulus ofiset and target onset. Each point in the figure represents the mean of the data for 5 subjects.

set-onset asynchrony $[F(7,28)=62.28, p<.01]$, as well as the interaction of location with offset-onset asynchrony $[F(7,28)=2.98, p<.05]$ were statistically significant. The analysis for manual latencies showed that the main effect of offset-onset asynchrony was statistically significant $[F(7,28)=90.20, p<.01]$.

The important conclusion that can be drawn from this experiment is that the patterns of results observed in Experiments 1 and 2 cannot be attributed to inappropriate allocation of attention. Importantly, saccadic latencies in the gap conditions were shorter when the unattended foveal stimulus was extinguished than when the attended eccentric stimulus was extinguished, even though the subjects were presented with a larger, more interesting, and conspicuous stimulus at the eccentric location.

\section{EXPERIMENT 4}

To remove any lingering doubt that the subjects were able to attend to the eccentric stimulus as instructed, a subsidiary measure was included in order to assess attentional allocation on each trial. As before, two stimuli were shown in the initial display: a foveal stimulus (a dot) located at the center of the display and an eccentric stimulus (a picture) located above or below the foveal stimulus. Both a dot and a picture were always present in the initial display. In some sessions the subjects were required to attend to the eccentric stimulus; in others they were required to attend to the foveal stimulus. The attentional condition, in which the subjects attended to the foveal stimulus, was included in the present experiment in order to permit us to evaluate the effects of direction of attention independently of the effects of the location of the offset.

Attentional allocation was measured by requiring the subjects to respond manually to stimulus offset, which could be foveal or eccentric. We would expect that in sessions in which the subjects were instructed to attend to the foveal stimulus, manual latencies would be shorter to the offset of the foveal stimulus than to the offset of the eccentric stimulus. Similarly, in sessions in which the subjects were instructed to attend to the eccentric stimulus, manual latencies should be shorter to the offset of the eccentric stimulus (Eriksen \& Hoffman, 1972; Posner et al., 1980). This would indicate that the subjects were attending as instructed.

It is important to note that in the manual task of the present experiment, the subjects responded to the offset of the stimulus at the foveal or the eccentric location. Thus, on every trial except catch trials, the subjects were required to execute a manual response to the offset of the foveal or the offset of the eccentric stimulus. In the previous experiments, the manual response was to the onset of the target.

The key question was whether the pattern of saccadic latencies in the present experiment would continue to be the same as in the previous experiments, in which attentional allocation was not assessed directly.

\section{Method}

Subjects. Five subjects, including the two authors and 3 paid subjects who were naive as to the experimental hypotheses, participated in the experiment. The naive subjects were recruited from a nearby university community and all were experienced psychophysical observers. Two of the naive subjects had participated in Experiment 3. The ages of the subjects ranged from 25 to 40 years.

Stimuli and Apparatus. The stimuli, their arrangement on the screen, and the apparatus were identical to those in Experiment 3.

Design and Procedure. There were three factors-direction of attention (foveal or eccentric), location of offset (foveal or eccentric), and offset-onset asynchrony $(0,100,150,200$, and $300 \mathrm{msec}$ )-for a total of 20 experimental conditions. Each condition was tested 40 times in the experiment. The direction of attention (foveal or eccentric) was held constant for a given session and the other two factors were varied randomly within a session. Thus, 
there were two types of sessions: attend-foveal and attend-eccentric. Each subject served in 10 sessions of each type, with 40 trials per session. In each session, there were an additional 16 catch trials, which are described below. One attend-foveal and one attendeccentric session were completed during the same sitting. One or two sittings were completed per day. The entire experiment was completed over the course of 1-2 weeks.

At the beginning of each trial, a dot and a picture appeared on the screen. The subjects foveated the central dot and, depending on the session, focused their attention on either the foveal dot or the eccentric picture, which appeared above or below the central dot. The subjects initiated each trial with a buttonpress when they felt confident that they were attending properly. The trial began after a random interval of between 0.9 and $1.9 \mathrm{sec}$. The subjects pressed a button as quickly as possible when they perceived the offset of the foveal dot or the offset of the eccentric picture. As before, the target appeared $3^{\circ}$ to the left or to the right of fixation and the subjects executed a saccade to the target as quickly as possible.

Catch trials were also included in the experiment. In these trials, an offset did not occur but the target still appeared. The subjects continued to make a saccade to the onset of the target, but had to withhold a manual response. The purpose of the catch trials was to ensure that, in the manual task, the subjects responded to the offset of the foveal or the eccentric stimulus and not to the onset of the target. The subjects were alerted to an incorrect manual response with a beep and the trial was repeated later in the same session. As mentioned above, there were 16 catch trials per session. With reference to the saccadic response, the catch trials were equivalent to the overlap conditions of the previous experiments because the saccadic target appeared when both stimuli in the initial display were still present.

Rejected trials. Trials were rejected and replaced in the same session if there was a problem with the saccadic or manual response. A problem with a saccade was identified if its latency fell outside the range of $100-600 \mathrm{msec}$, if the saccade was in the wrong direction, or if fixation was not stable during the initial stimulus display. A problem with a manual response was identified if its latency fell outside the range of $150-650 \mathrm{msec}$ or if the manual response occurred on a catch trial. On the average, $10 \%$ of the trials were rejected during a session. The majority of rejections occurred because (1) a manual response occurred on a catch trial (3\% of trials) or (2) a manual response was excessively delayed to the unattended stimulus ( $3 \%$ of trials).

\section{Results and Discussion}

Figure 4 shows the results of the present experiment, averaged over all 5 subjects. Saccadic latencies are shown in the top two panels, and manual latencies are shown in the bottom two panels. Results from the sessions in which the subjects attended toward the eccentric stimulus are shown on the left, and results from the sessions in which they attended toward the foveal stimulus are shown on the right.

Manual latencies. The purpose of the manual task was to assess whether the subjects were able to direct their attention as instructed. We expected shorter manual latencies to the offset of the attended stimulus. The manual latencies
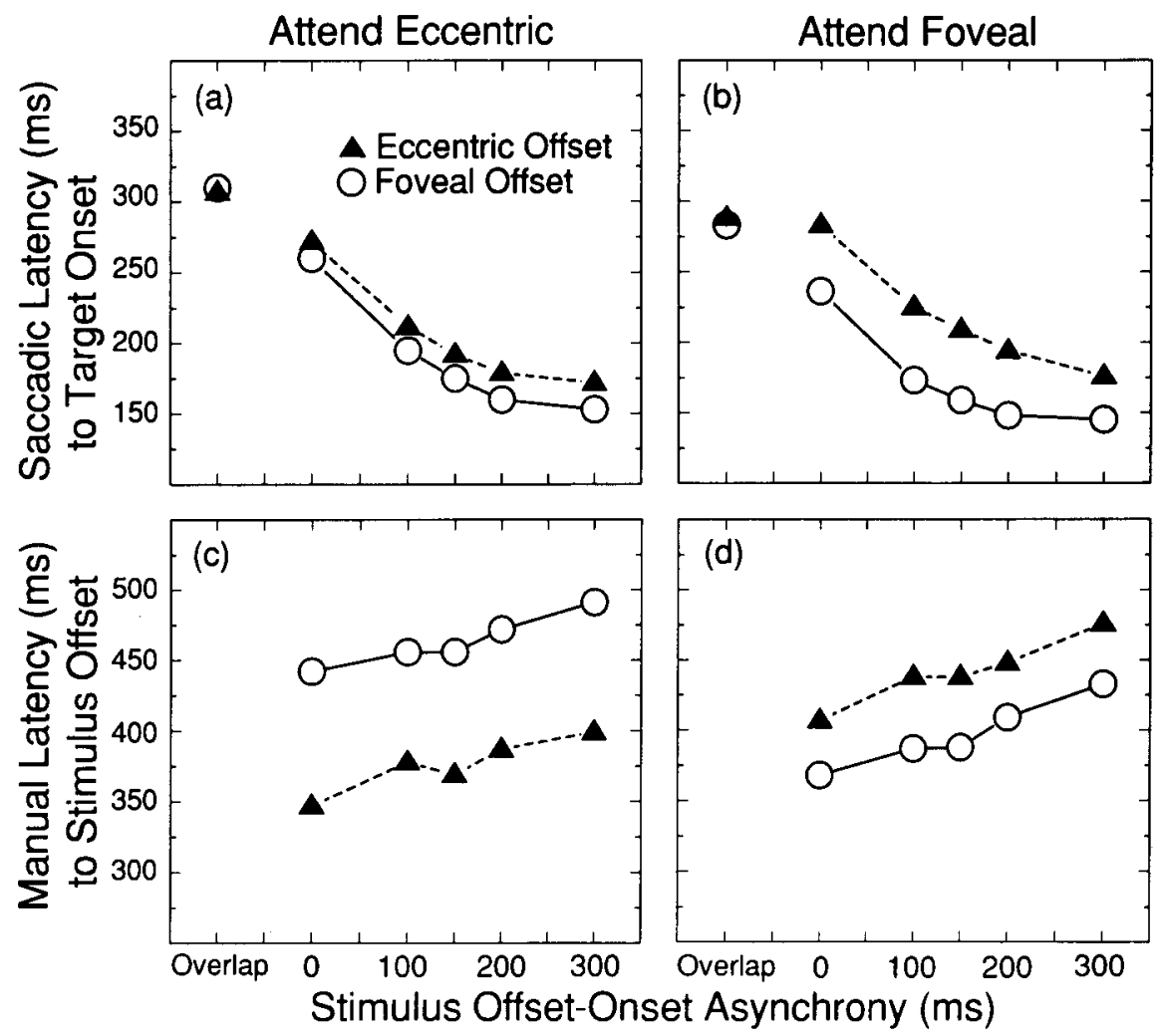

Figure 4. Saccadic, panels a and b, and manual, panels $c$ and d, latencies (from Experiment 4) are shown as a function of asynchrony between stimulus offset and target onset. Note that, in contrast to the previous figures, manual latencies were in response to stimulus offset and not to target onset. Each point in the figure represents the mean of the data for 5 subjects. 
indicated that the subjects were able to direct their attention toward the appropriate stimulus, as instructed on a given session; manual latencies to the offset of the attended stimulus were shorter than to the offset of the unattended stimulus (see bottom two panels of Figure 4). The curve corresponding to the attended stimulus lies below that corresponding to the unattended stimulus in both panels.

There was also an overall trend for manual latencies to increase with offset-onset asynchrony (see bottom two panels of Figure 4). This can be explained by assuming that, at short asynchronies, the manual response to stimulus offset was facilitated somewhat by target onset. Also, the separation of the curves was larger when the subjects attended to the eccentric stimulus than when they attended to the foveal stimulus, indicating that they found it easier to attend to the eccentric stimulus and to ignore the foveal stimulus than vice versa.

A 2 (direction of attention) $\times 2$ (location of offset) $\times$ 5 (offset-onset asynchrony) within-subject analysis of variance showed that, for the manual latencies, the interaction of direction of attention and location of offset was statistically significant $[F(1,4)=112.15, p<.01]$. This reflects the fact that the curve for the attended offset was always below the curve for the unattended offset; filled triangles are below the open circles in Figure $4 c$, whereas open circles are below the filled triangles in Figure $4 \mathrm{~d}$. The main effect of asynchrony $[F(4,16)=6.04, p<.01]$ and the main effect of location of offset $[F(1,4)=9.73, p<.05]$ were also statistically significant. This latter effect indicated that manual latencies were, on the average, shorter to the eccentric offset than to the foveal offset. Other effects were not statistically significant.

Saccadic latencies. Saccadic latencies are shown in the top two panels of Figure 4. For saccadic latencies, the results replicated those of Experiment 3 . In gap conditions, latencies were shorter when the foveal stimulus was extinguished than when the eccentric stimulus was extinguished. This was true in both attentional conditions, when attention was directed toward the eccentric stimulus (Figure 4a) and when it was directed toward the foveal stimulus (Figure $4 b$ ). Notably, saccadic latencies were shorter in the foveal-offset condition, even when the foveal stimulus was unattended (Figure 4a).

This dominant foveal-offset effect on saccadic latencies was modulated by attention. The effect of attention was to shorten saccadic latencies for the attended offset and to lengthen latencies for the unattended offsets. Note that saccadic latencies for foveal offsets were shorter in the attend-foveal conditions compared with the attendeccentric conditions. Similarly, note that the saccadic latencies for the eccentric offset were shorter in the attendeccentric conditions than in the attend-foveal conditions. The net result was that the two curves in Figure 4a fall midway between the two curves in Figure $4 b$.

A 2 (direction of attention) $\times 2$ (location of offset) $\times$ 6 (offset-onset asynchrony) within-subject analysis of variance showed that, for the saccadic latencies, the main effects of location of offset $[F(1,4)=40.97, p<.01]$ and asynchrony $[F(5,20)=267.9, p<.01]$ were statistically significant.

All two-way interactions and the three-way interaction were statistically significant. In particular, the two-way interaction between direction of attention and location of offset was significant $[F(1,4)=10.71, p<.05]$. Note that the filled triangles and the open circles lie closer together in Figure 4a than in Figure $4 b$, indicating that attention modulated but did not reverse the effect of location of offset. A significant two-way interaction between location of offset and offset-onset asynchrony is also reflected in the graphs $[F(5,20)=17.63, p<.01]$. The filled triangles and the open circles are superimposed in the overlap conditions, but are separated in the gap conditions. Finally, the two-way interaction between direction of attention and offset-onset asynchrony was also significant $[F(5,20)=14.5, p<.01]$.

The three-way interaction of direction of attention, location of offset, and offset-onset asynchrony was statistically significant $[F(5,20)=3.32, p<.05]$. This threeway interaction is observed in the top panels of Figure 4 in that, in overlap conditions, the triangles and circles are superimposed in both the left and right panels, but in gap conditions the separation between the two curves are smaller in the left (Figure 4a) than in the right panel (Figure $4 \mathrm{~b}$ ). Planned comparisons showed that the difference between the two curves at each offset-onset asynchrony between 0 and $300 \mathrm{msec}$ in Figure 4a (attend-eccentric conditions), although reduced, were significant at least at the $p<.05$ level.

In summary, a saccade could be executed more rapidly when the foveal stimulus was extinguished before the onset of the target than when the eccentric stimulus was extinguished. This effect was modulated but not reversed by directed attention.

\section{EXPERIMENT 5}

This experiment was motivated by issues surrounding express saccades. Fischer, Ramsperger, Weber, and colleagues have observed that in gap conditions, saccadic latencies can be extremely short, down to about $100 \mathrm{msec}$ (e.g., Fischer \& Ramsperger, 1984; Fischer \& Weber, in press). Furthermore, saccadic latencies may segregate into two distributions, one centered at about $120 \mathrm{msec}$ and the other centered at about $150 \mathrm{msec}$. Those in the distribution centered around $120 \mathrm{msec}$ are referred to as express saccades; those in the other distribution are referred to as fast regular. It has been suggested that express saccades occur only when attention is disengaged prior to the saccade (e.g., Braun \& Breitmeyer, 1988; Mayfrank et al., 1986). This would lead us to expect two distributions of saccades when the attended stimulus is extinguished prior to target onset because, as described above, turning off the attended stimulus is believed to disengage attention (e.g., Fischer, 1987; Braun \& Breitmeyer, 1988).

In order to evaluate the qualitative aspects of the latency distributions and determine whether there existed 
separate populations of saccades, the video-based eyemovement monitor used in the previous experiments was supplemented with an EOG (electrooculograph) system. The sampling rate of the video-based system $(60 \mathrm{~Hz})$ was inadequate to discriminate two distributions separated by $30 \mathrm{msec}$. The sampling rate of the EOG-based system was set to $4 \mathrm{KHz}$, thus providing ample temporal resolution to identify separate populations of saccades.

\section{Method}

Subjects. The two authors served as subjects in the experiment.

Stimuli and Apparatus. The stimuli, their arrangement on the screen, and the apparatus were identical to those in Experiments 3 and 4 , except that the target appeared $5^{\circ}$ to the left or to the right of fixation.

In addition to the ISCAN RK-416 eye-movement monitoring system, an EOG-based eye-movement monitoring system was also used. Three electrodes were attached to each subject: one to the outer canthus of each eye and one common electrode to the forehead. The output from the electrodes was fed into two Tektronix 502 differential amplifiers, one for the left and one for the right eye. The amplifiers were set to lowpass filter the signal and to boost the gain by $10^{5}$. The signal was subsequently digitized with a precision of 12 bits at $4 \mathrm{KHz}$ by a Data Translation AD2821 A/D board. The gain on the input of the A/D board was set to match the amplitude of the signals from the two eyes.

Saccades were detected by searching for the maximum of the second derivative of the EOG signal. The maximum of the second derivative corresponds to the point at which the EOG trace just begins to depart from its baseline value. This was considered to be the onset of a saccade and was verified by comparing it to the value obtained from the ISCAN system. The EOG and ISCAN estimates had to agree to within $\pm 16.7 \mathrm{msec}$, which is the uncertainty of the ISCAN measurement, or the trial was rejected and replaced later in the session.

Design and Procedure. The design was the same as that in Experiment 4 , except that each subject completed 60 rather than 20 sessions ( 30 sessions were attend-foveal and 30 sessions were attend-eccentric).

Rejected trials. Trials were rejected and replaced in the same session if there was a problem with the saccadic or manual response, as described in Experiment 4. In addition, trials were replaced if measurements of saccadic latencies with the video-based system and with the EOG-based system differed by $16.7 \mathrm{msec}$ or more. On the average, the percentage of trials rejected during a session was $12 \%$ for W.J.T. and $20 \%$ for L.B.S. The majority of rejections occurred because (1) measurements with the ISCAN and EOG systems differed on $6 \%$ and $7 \%$ of the trials for W.J.T. and L.B.S., respectively, or (2) a manual response was made on a catch trial on $2 \%$ and $7 \%$ of the trials for W.J.T. and L.B.S., respectively. Discrepancies between the ISCAN and the EOG measurements were primarily due to noise in the EOG signal.

\section{Results and Discussion}

Frequencies of saccadic latencies for the gap conditions $(100,150,200$, and $300 \mathrm{msec})$ were pooled and are shown in Figure 5. The data for Subject W.J.T. are shown in the top two panels and those for Subject L.B.S. are shown in the bottom two panels. The unfilled bars represent the case in which the foveal stimulus was turned off, and the filled bars represent the case in which the eccentric stim-

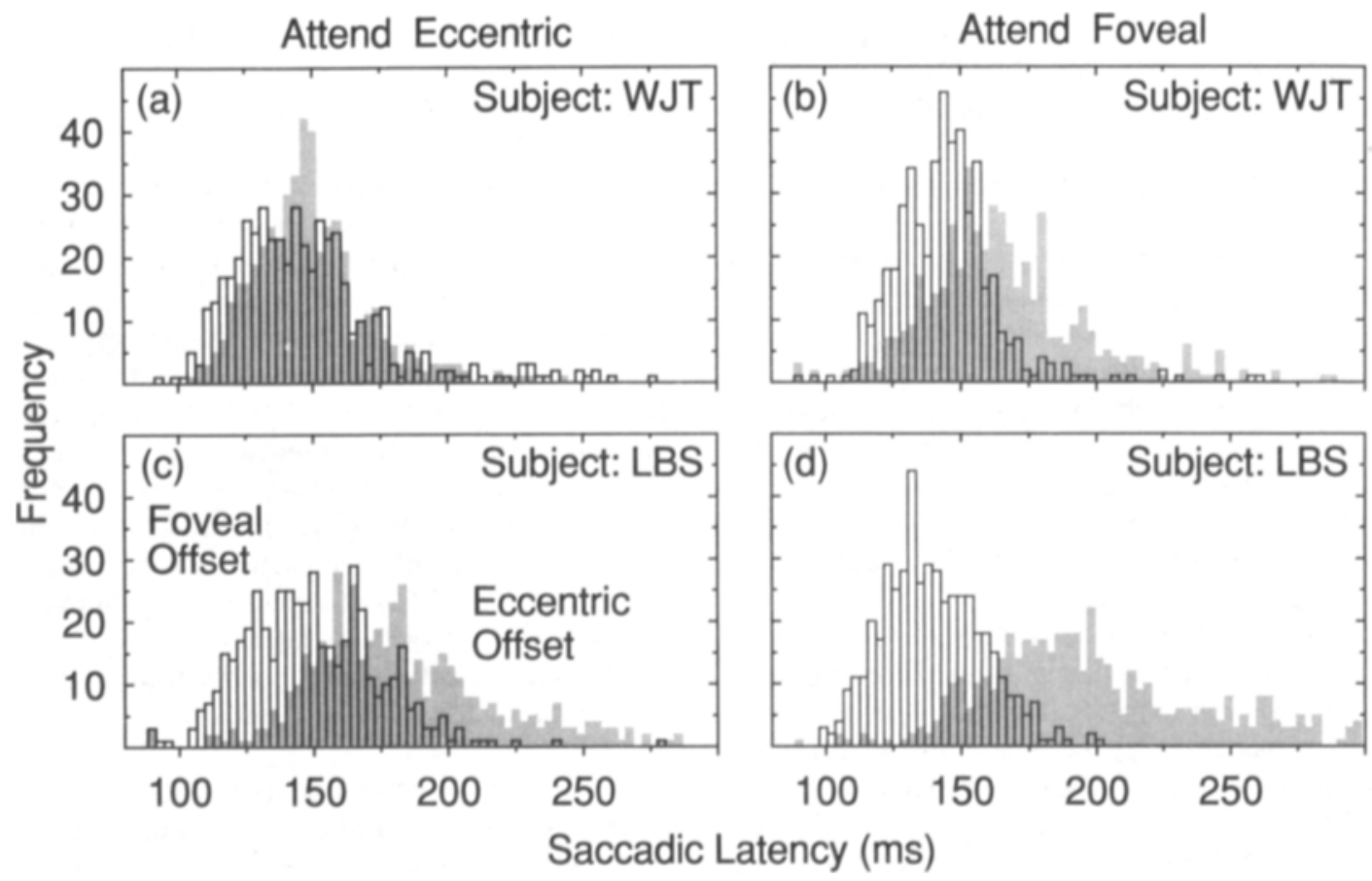

Figure 5. Frequency distributions of saccadic latencies for Subjects W.J.T., panels a and b, and L.B.S., panels c and $\mathbf{d}$ (from Experiment 5). The unfilled bars represent latencies obtained with foveal offsets and the filled bars represent latencies obtained with eccentric offsets. Each histogram consists of 480 trials and is based on the pooled data for gap conditions (100-, 150-, 200-, and 300-msec offset-onset asynchronies). 
ulus was turned off. The panels on the left represent the attend-eccentric condition, and the panels on the right represent the attend-foveal condition.

Bimodal distributions in the saccadic latency histograms, with one peak at $120 \mathrm{msec}$ and another at $150 \mathrm{msec}$, were evident in one case-for Subject W.J.T. for the unfilled bars in Figure 5b. There was also an indication of two distributions for Subject L.B.S. for the unfilled bars in Figure $5 \mathrm{c}$. In both cases, signs of bimodality were evident only in foveal-offset conditions. The occurrence of bimodal distributions was not reliably associated with attended offsets, as would be expected if attentional disengagement gave rise to express saccades.

Irrespective of the occurrence of bimodal distributions, it is clear that there were more short-latency saccades for the foveal-offset conditions, regardless of attentional allocation. This is evident in that the unfilled bars lie to the left of the filled bars in Figure 5. These results are consistent with those of Experiment 4, which showed that latencies were shorter when the foveal stimulus was extinguished, even when attention was focused eccentrically. Similar results have also been recently reported by Klein, Kingstone, and Pontefract (1992).

Many of the short-latency saccades in the foveal-offset conditions fell within the range of express saccades $(90-$ $135 \mathrm{msec}$ ). This is not what would be expected if attention determined the occurrence of express saccades; one would expect more express saccades in the attended-offset conditions. Instead, more express saccades were observed in the foveal-offset conditions, regardless of the direction of attention. Clearly, another explanation is needed, possibly one that uses ocular disengagement rather than attentional disengagement to explain the observed effects.

\section{GENERAL DISCUSSION}

The goal of the present work was to compare and examine the contribution of the attentional and ocular sampling systems to saccadic latencies. The unique aspect of the present research is that the two systems were studied together, whereas in previous work they were studied separately. Three possibilities were considered: saccadic latencies are determined by the ocular sampling system, by the attentional sampling system, or jointly by both systems. These possibilities were examined in a series of five experiments.

The important aspects of the results were as follows. First, turning off either the foveal or the eccentric stimulus resulted in shorter saccadic latencies. Second, turning off the foveal stimulus resulted in shorter saccadic latencies than turning off the eccentric stimulus. Third, direction of attention modulated, but did not reverse, this latter effect; saccadic latencies to the target continued to be shorter in the foveal-offset condition than in the eccentric-offset condition, regardless of the direction of attention. Fourth, express saccades were more prominent with foveal offsets than with eccentric offsets, regardless of the direction of attention.

In the next section we will consider three possible explanations for the results: (1) an ocular explanation, (2) an attentional explanation, and (3) a joint ocular and attentional explanation.

\section{An Ocular Explanation}

The patterns of results observed with saccadic latencies can be explained with reference to the ocular sampling system. According to this explanation, the ocular system is engaged when a stimulus is foveated. In turn, the foveal stimulus helps to maintain the ocular system in an engaged state. A saccade to a new stimulus must be preceded by disengagement. Disengagement can be facilitated by turning off the stimulus at fixation. Hence, in the present experiments, extinguishing the foveal stimulus resulted in shorter saccadic latencies because of facilitation of ocular disengagement. Longer saccadic latencies were observed when the eccentric stimulus was extinguished, presumably because ocular disengagement was not facilitated to the same degree. That is, the subjects began to disengage fixation when they detected the offset of the eccentric stimulus, but disengagement took more time because the foveal stimulus was still present.

The results were consistent with this explanation, with the exception that it did not explain why direction of attention had a modulating effect on saccadic latencies. This modulating effect was to produce shorter saccadic latencies for attended than for unattended offsets (see top two panels in Figure 4). The modulating effect of directed attention can be explained by assuming that attention affected the detection of the foveal and the eccentric offset. The offset at the focus of attention would be detected sooner, thus leading to shorter latencies (e.g., Stelmach \& Herdman, 1991).

\section{An Attentional Explanation}

It is not possible to explain the present results with reference only to the attentional sampling system. The attentional explanation assumes that attention is engaged when a stimulus is attended and that a saccade to a new stimulus must be preceded by attentional disengagement. As generally assumed, attentional disengagement can be facilitated by turning off the attended stimulus (Braun \& Breitmeyer, 1988; Fischer, 1987; Mayfrank et al., 1986). According to this explanation, saccadic latencies to a target should be shorter when an attended stimulus is extinguished than when an unattended stimulus is extinguished. The results of the present work did not support these expectations. The difficult aspect for the attentional explanation is that the saccadic latencies for the foveal offsets were always shorter than those for the eccentric offsets, regardless of direction of attention.

\section{An Ocular-Attentional Explanation}

There exists yet a third possibility - that engagement and disengagement of both the ocular and attentional systems play a role in determining saccadic latencies. According to this explanation, saccadic latencies would be determined by the system that takes longer to disengage. If one system is already disengaged, then latencies would be determined by the system that is still engaged. Disengagement of either system could proceed in parallel. 
The present data can be explained by assuming that (1) turning off the attended stimulus promoted disengagement of the attentional system and that turning off the foveal stimulus promoted disengagement of the ocular system, and (2) that attentional disengagement was faster than ocular disengagement. This latter assumption is consistent with the view that eye movements are preceded by attentional shifts to the saccadic target (Henderson, Pollatsek, \& Rayner, 1989; Posner, 1980; Shepherd et al., 1986).

Consider the four curves in the top panels of Figure 4. The shortest saccadic latencies would be expected when both systems are disengaged. This occurred when the attended foveal stimulus was extinguished (Figure $4 b$, open circles). The next shortest saccadic latencies would be expected when the slower ocular system was already disengaged and the faster attentional system remained to be disengaged. This occurred when attention was directed eccentrically and the foveal stimulus was extinguished (Figure $4 a$, open circles). The slowest saccadic latencies would be expected when the slower ocular system remained to be disengaged. This $\alpha-$ curred when the eccentric stimulus was extinguished and attention was directed either eccentrically (Figure $4 a$, filled triangles) or foveally (Figure $4 \mathrm{~b}$, filled triangles).

The results are consistent with the ocular-attentional explanation, with one exception. This explanation would predict that the filled triangles in Figure 4a should coincide with the filled triangles in Figure $4 \mathrm{~b}$. In fact, the attended eccentric offset produced slightly shorter saccadic latencies than the unattended eccentric offset. Specific comparisons showed that the filled triangles in Figure 4a were below the filled triangles in Figure $4 \mathrm{~b}$ at offset-onset asynchronies of $0,100,150$, and $200 \mathrm{msec}$ at least at the $p<.05$ level. This difference is inconsistent with the ocular-attentional explanation. It could be attributed to more ready detection of the attended offset, as mentioned above, or to a cost associated with the need to simultaneously disengage the two systems.

For all three explanations considered here, it is important to bear in mind that general alerting due to the foveal or eccentric offsets may have played a role in shortening saccadic latencies, over and above the effects of ocular disengagement, attentional disengagement, and attentional modulation (e.g., Ross \& Ross, 1980, 1981). An effect of alerting was suggested by the manual latencies in Experiments 2 and 3; manual latencies to the onset of the target were shorter in gap than in overlap conditions, as discussed in Experiment 2.

In summary, three possible explanations for the present data were considered: an ocular, an attentional, and an ocular-attentional explanation. It was concluded that the ocular and the ocular-attentional explanations provided a satisfactory account for the present data, but that the attentional explanation failed to account for the data. Of the two explanations that were successful, the ocular explanation was simpler. Nonetheless, it may still be premature to discount a role for the ocular-attentional explanation in accounting for saccadic latencies in displays such as those used in the present research. This remains a topic for future research.

\section{REFERENCES}

BECKER, W. (1972). The control of eye movements in the saccadic system. Bibliotheca Ophthalmologica, 82, 233-243.

BRAUN, D. , BREITMEYER, B. G. (1988). Relationship between directed visual attention and saccadic reaction times. Experimental Brain Research, 73, 546-552.

Cohen, M. E., \& Ross, L. E. (1977). Saccadic latency in children and adults: Effects of warning interval and target eccentricity. Joumal of Experimental Child Psychology, 23, 539-549.

ENGEL, R. L. (1971). Visual conspicuity, directed attention and retinal locus. Vision Research, 11, 563-576.

Eriksen, C. W., HoffMan, J. E. (1972). Temporal and spatial characteristics of selective encoding from visual displays. Perception \& Psychophysics, 12, 201-204.

FindLaY, J. (1981). Spatial and temporal factors in the predictive generation of saccadic eye movements. Vision Research, 21, 347-354.

Finley, G. (1985). A high-speed point plotter for vision research. Vision Research, 25, 1993-1997.

Fischer, B. (1987). The preparation of visually guided saccades. Review of Physiology, Biochemistry, \& Pharmacology, 106, 1-35

FisCHER, B., \& BOCH, R. (1983). Saccadic eye movements after extremely short reaction times in the monkey. Brain Research, 260 , 21-26.

FisChER, B., \& BreITMEYer, B. (1987). Mechanisms of visual attention revealed by saccadic eye movements. Neuropsychologia, 25 , 73-78.

Fischer, B., \& RAMSPERGER, E. (1984). Human express saccades: Extremely short reaction times of goal directed eye movements. Experimental Brain Research, 57, 191-195.

FISCHER, B., \& WEBER, H. (in press). Express saccades and visual attention. Behavioral \& Brain Sciences.

Groner, R., \& Groner, M. T. (1989). Attention and eye movement control: An overview. European Archives of Psychiatry \& Neurological Sciences, 239, 9-16.

Hallett, P. E., \& Adams, B. D. (1980). The predictability of saccadic latency in a novel voluntary oculomotor task. Vision Research, 20, 329-339.

Henderson, J. M., Pollatsek, A., \& Rayner, K. (1989). Covert visual attention and extrafoveal information use during object identification. Perception \& Psychophysics, 45, 196-208.

JoNiDES, J. (1980). Towards a model of the mind's eye's movement. Canadian Journal of Psychology, 34, 103-112.

Kalesnykas, R. P., \& Hallett, P. E. (1987). On plotting amplitudetransition functions for voluntary eye saccades. Vision Research, 27, 675-679.

KaufMaN, L., \& Richards, W. (1969). Spontaneous fixation tendencies for visual forms. Perception \& Psychophysics, 5, 85-88.

KLEIN, R. (1980). Does oculomotor readiness mediate cognitive control of visual attention? In R. S. Nickerson (Ed.), Attention and performance VII (pp. 259-275). Hillsdale, NJ: Erlbaum.

Klein, R., Kingstone, A., \& Pontefract, A. (1992). Orienting of visual attention. In K. Rayner (Ed.), Eye movements and visual cognition (pp. 46-65). New York: Springer-Verlag.

LABERGE, D., \& Brown, V. (1989). Theory of attentional operations in shape identification. Psychological Review, 96, 101-124.

Mayfrank, L., Mobashery, M., Kimmig, H., \& Fischer, B. (1986). The role of fixation and visual attention in the occurrence of express saccades in man. European Archives of Psychiatry \& Neurological Sciences, 235, 269-275.

PoSNER, M. I. (1980). Orienting of attention. Quarterly Journal of Experimental Psychology, 32, 3-25.

Posner, M. I., Snyder, C. R. R., \& Davidson, B. J. (1980). Attention and the detection of signals. Journal of Experimental Psychology: General, 109, 160-174.

Remington, R. W. (1980). Attention and saccadic eye movements. 
Journal of Experimental Psychology: Human Perception \& Performance, 6, 726-744.

Reulen, J. P. H. (1984). Latency of visually evoked saccadic eye movements: 1. Saccadic latency and the facilitation model. Biological Cybernetics, 50, 251-262.

Reuter-Lorenz, P. A., Hughes, H. C., \& Fendrich, R. (1991). The reduction of saccadic latency by prior offset of the fixation point: An analysis of the gap effect. Perception \& Psychophysics, 49, 167-175.

Ross, L., \& Ross, S. (1980). Saccade latency and waming signals: Stimulus onset, offset, and change as warning events. Perception \& Psychophysics, 27, 251-257.

Ross, S., \& Ross, L. (1981). Saccade latency and warning signals: Effects of auditory and visual stimulus onset and offset. Perception \& Psychophysics, 29, 429-437.

Ross, S., \& Ross, L. (1983). The effects of onset and offset warning and post-target stimuli on the saccade latency of children and adults. Journal of Experimental Child Psychology, 36, 340-355.

SASLOW, M. G. (1967). Effects of components of displacement-step stimuli upon latency for saccadic eye movements. Journal of the Optical Society of America, 57, 1024-1029.
ShePherd, M., Findlay, J. M., \& Hockey, R. J. (1986). The relationship between eye movements and spatial attention. Quarterly Journal of Experimental Psychology, 38A, 475-491.

Stelmach, L. B., Herdman, C. M. (1991). Directed attention and perception of temporal order. Journal of Experimental Psychology: Human Perception \& Performance, 17, 539-550.

TAM, W. J. (1989). Saccadic latency and an oculomotor unlocking process. Unpublished doctoral dissertation, York University, Toronto, Canada.

TAM, W. J., \& ONO, H. (1984). Reduction in saccadic and vergence latencies as a function of time between fixation stimulus offset and target onset. Investigative Ophthalmology \& Visual Science, 25(Suppl.), 182.

TAM, W. J., \& ONO, H. (1988). Visual fixation, pursuit and saccades. Investigative Ophthalmology \& Visual Science, 29(Suppl.), 346.

Wenban-Smith, M. G., \& Findlay, J. M. (1991). Express saccades: Is there a separate population in humans? Experimental Brain Research, 87, 218-222.

(Manuscript received March 27, 1992; revision accepted for publication January 27, 1993.) 